\title{
INFLUENCE OF DRUG-RESISTANT EPILEPSY ON SURGICAL CARE OF DEEP BURN INJURY
}

M.D. Ćertić Biljana, Specialist for plastic and Reconstructive Surgery

Clinic for Burns, Plastic and Reconstructive Surgery, Clinical Center of Serbia, Belgrade

Epilepsy is a neurological disorder marked by sudden recurrent episodes of sensory disturbance, loss of consciousnes or convulsions, associated with abnormal electrical activity in the brain. Majority of epileptic patients tend to present with accidental deep contact burns usually induced by flame or hot water.
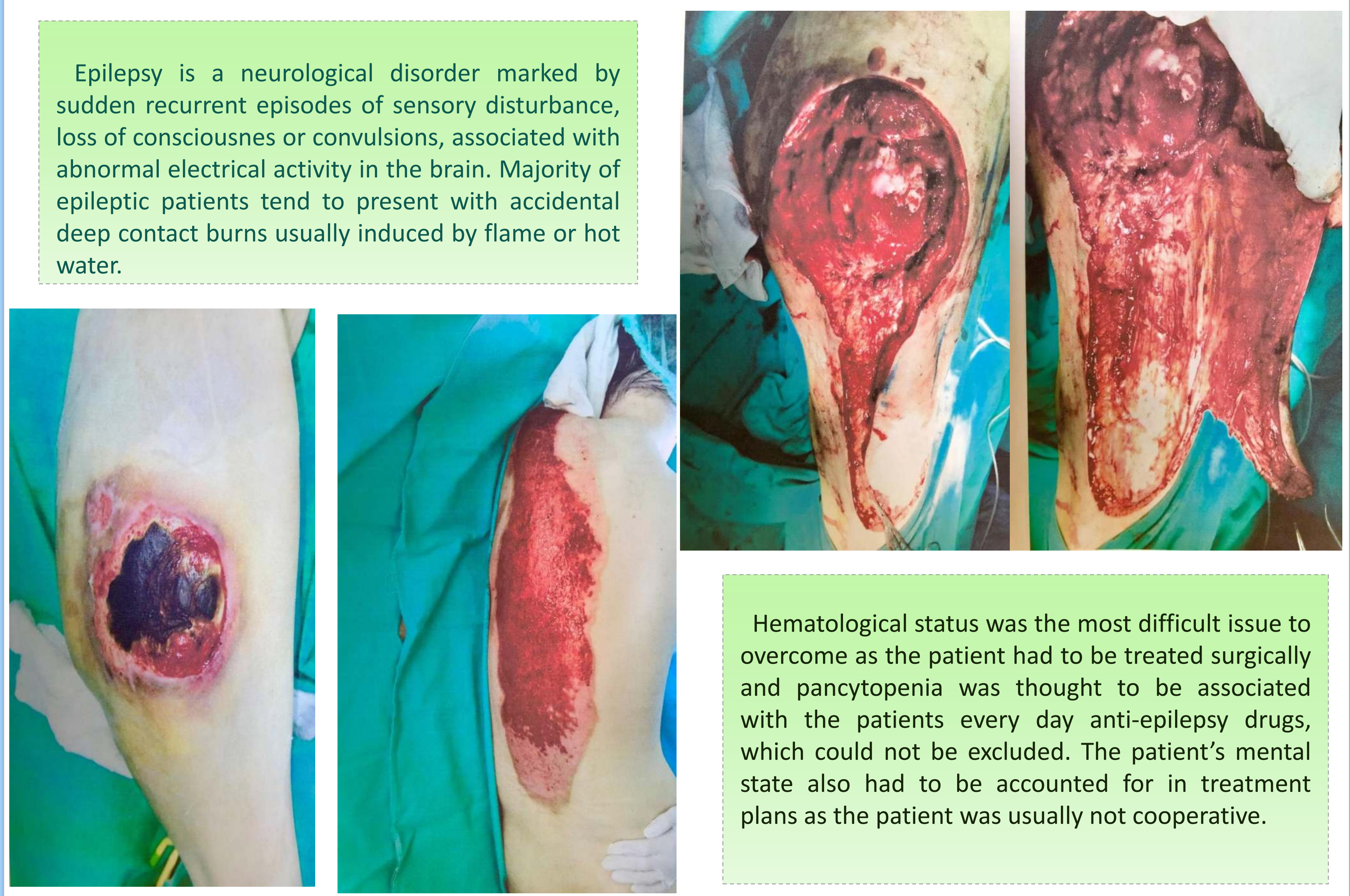

This poster prents the case of a 40 year old man patient with drug-resistant epilepsy who was admitet to our hospital with a deep $2^{\text {nd }}$ and $3^{\text {rd }}$ degree burn injury of $7 \%$ TBSA. Multiple comorbidites presented on various organs and systems as he suffered from temporal lobe glioma, pancytopenia, erosive gastritis and eventualy cachexia and decubital wounds.

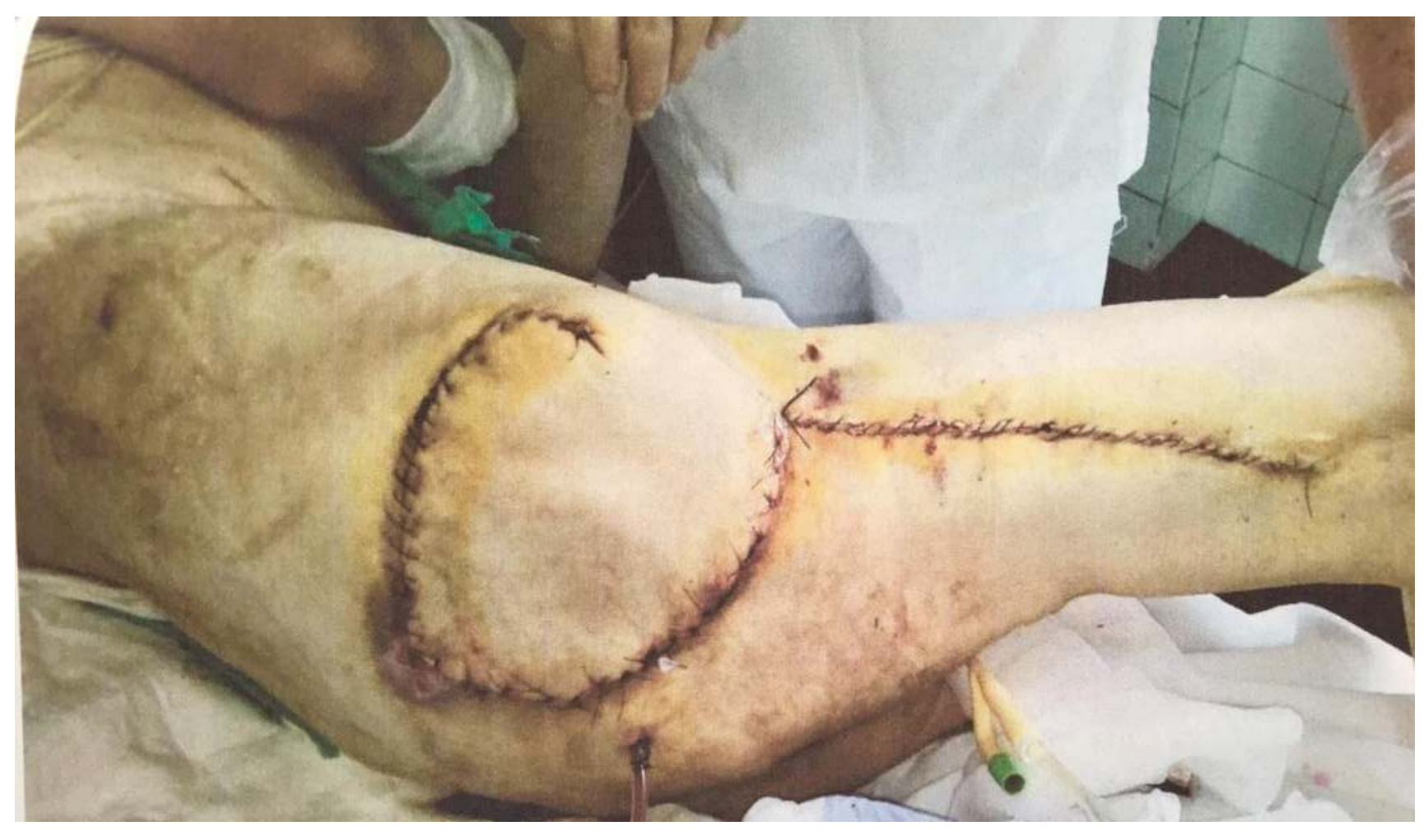

Hematological status was the most difficult issue to overcome as the patient had to be treated surgically and pancytopenia was thought to be associated with the patients every day anti-epilepsy drugs, which could not be excluded. The patient's mental state also had to be accounted for in treatment plans as the patient was usually not cooperative.

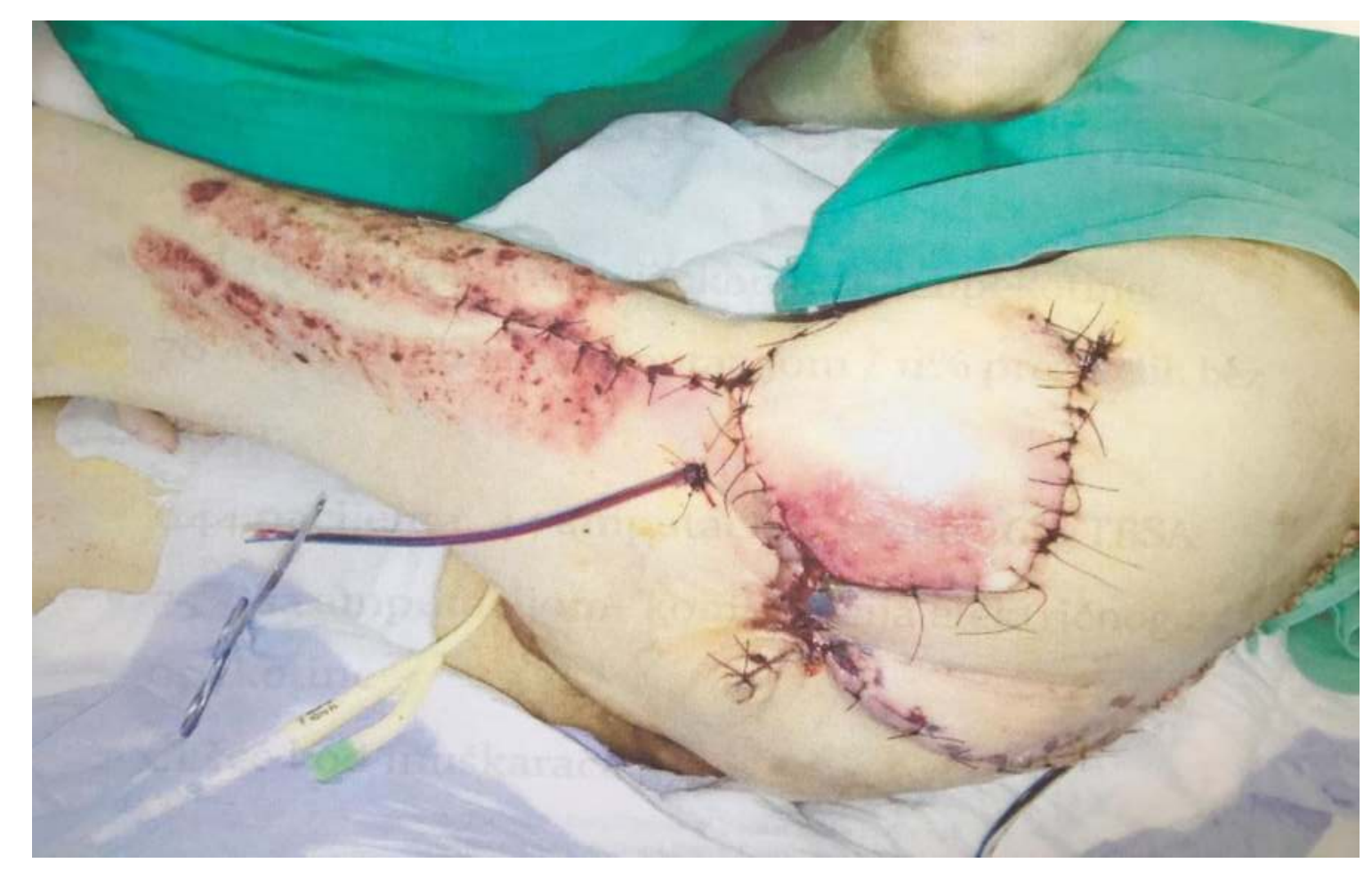

\section{Conclusion}

Accodental burn prevention should be advocated to severe epilepsy patients and especially their families, in an attempt to avoid burns in this group, as their care and treatment can be very challenging due to the fact of potential comorbidities which can hamper the success of surgical treatment. 